\title{
COMPORTAMENTO DE EXTRATORES EM SOLO TRATADO COM FONTES DIVERSAS DE FÓSFORO'
}

\author{
R. STEFANUTTI; T. MURAOKA; E. MALAVOLTA \\ Centro de Energia Nuclear na Agricultura - USP - C.P. 96 - CEP: 13400-970 - Piracicaba,SP
}

\begin{abstract}
RESUMO: Em condiç̄es de casa-de-vegetaçăo foram feitos sete cultivos sucessivos para avaliar o efeito direto e residual do superfosfato simples granulado e do termofosfato magnesiano (Yoorin) fornecidos em quatro doses $(0$, 50,100 e $200 \mathrm{ppm}$ ). O fosforo disponfivel foi determinado depois do terceiro, quarto e filtimo cultivos usando-se os extratores de Mehlich 1, Bray 1, resina e a técnica de diluị̧̧ăo isotópica (valor E). De um modo geral, os coeficientes de correlação mais altos entre $\mathbf{P}$ disponível e matéria seca ou fosforo total absorvido, foram encontrados com os dados obtidos com resina e Mehlich 1, seguindo-se os obtidos com Bray 1 e, em uiltimo lugar, os com o valor $\mathbf{E}$. Descritones: adubos fosfatados, extratores, fosforo disponivel
\end{abstract}

\section{PERTORMANCE OR EXTRACTING PROCEDURES IN SOIL TREATED WITH SEVERAL SOURCES OF PHOSPHORUS}

\begin{abstract}
ARSTRACT: In order to evaluate the direct and residnal effects of ordinary granulated superphosphate as compared to magnesium thermophosphate (Yoorin), supplied at four rates $(0,50,100$ and $200 \mathrm{ppm})$, seven successive croppings were carried out in the glasshouse. Available phosphorus was assessed after the third, fourth and seventh harvest by using Mehlich 1, Bray 1, anion exchange resin and isotopic dilution ( $E$ value). The highest correlation coefficients between available $P$ and either total dry matter or total $P$ content was found to occur with data provided by Mehlich 1 and resin. Phosphorus extracted by Bray 1 as well as that estimated by the $E$ value showed lower " $r$ " coefficients. Key Words: phosphate fertilizers; extracting agents; available phosphorus
\end{abstract}

\section{INTRODUÇÃO}

E relativamente grande o número de métodos de extração do fósforo, os quais tem $\mathrm{em}$ comum, o objetivo de simular no laboratório o que a raiz faz no campo. Esses métodos fazem uso de soluções diluídas de ácidos minerais, salinas e de resina trocadora de íons ou do princípio da diluição isotópica (RAIJ, 1978, ANGHINONI \& VOLKWEISS, 1984). O extrator de Mehlich número 1, ainda o mais usado no Brasil, é recomendado para solos ácidos, de baixa capacidade de troca catiônica (CTC) e com predominância de caolinita e sesquióxidos na fração argila (KAMPRATH \& WATSON, 1980). O extrator de Bray-1 contém ácido clorídrico diluído e fluoreto de amônio que complexa o cálcio, o alumínio e o ferro liberando o P (SMITH et al., 1957). Através da diluição isotópica entre o fosfato ${ }^{31} \mathrm{P}$ do solo $\mathrm{e}$ o fosfato ${ }_{-32} \mathrm{P}$ adicionado pode-se estimar o teor do primeiro que corresponde ao existente na solução e mais a forma "lábil" (McAULIFFE et al., 1948). A resina deve extrair as mesmas formas (RAIJ et al., 1986).

As plantas adubadas absorvem além do fósforo nativo, produtos da transformação do $\mathrm{P}$ do fertilizante no solo. Tais produtos dependem dos constituintes do adubo e das características químicas do solo - $\mathrm{pH}$, teores de cálcio, ferro, alumínio, principalmente. Segue-se daí que, por sua natureza, a eficiência do extrator, ou o seu poder de discriminação, poderá depender, num mesmo solo, da fonte de P empregada. É esta a hipótese do presente trabalho que compara extratores do $\mathbf{P}$ do solo resultante da aplicação de dois adubos fosfatados, o superfosfato simples (SPS) e o termofosfato magnesiano (TF), este em diferentes granulometrias.

\section{MATERIAL \& METODOS}

No ensaio foram usados vasos de polietileno contendo $2 \mathrm{~kg}$ de terra, um Latossolo

\footnotetext{
1 Parte da dissertação de mestrado do primeiro autor apresentada ao CENA/USP, Piracicaba,SP.
} 
Vermelho Amarelo, fase cerrado, amostra colhida em Goiânia, GO, profundidade de $0-20 \mathrm{~cm}$, com as seguintes características: $\mathrm{pH}\left(\mathrm{H}_{2} \mathrm{O}\right)-4,6$; C \% - 1,1; P (Mehlich 1) - 1 ppm; K - 0,08 meq $/ 100 \mathrm{~cm}^{3} ; \mathrm{Ca}-0,4 \mathrm{meq} / 100 \mathrm{~cm}^{3} ; \mathrm{Mg}-0,3$ meq $/ 100 \mathrm{~cm}^{3} ; \mathrm{H}+\mathrm{Al}-3,4 \mathrm{meq} / 100 \mathrm{~cm}^{3} ; \mathrm{S}^{-\mathrm{SO}_{4}}$ - $10 \mathrm{ppm}$; V - 22\%; areia $72 \%$; limo $7 \%$ e argila $21 \%$.

O solo foi corrigido com calcário dolomítico em dose calculada para elevar V\% a 60. $\mathrm{Em}$ todos os cultivos as plantas receberam $150 \mathrm{ppm}$ $\mathrm{N}$ e $150 \mathrm{ppm}$ de $\mathrm{K}$ em três aplicações iguais, a primeira no plantio e as demais em cobertura, exceto no primeiro cultivo com feijão mungo $\mathrm{em}$ que se usou apenas a dose inicial.

Empregou-se o termofosfato magnesiano (Yoorin) em três granulometrias: pó (100\% passando por peneira de malha de $0,84 \mathrm{~mm}$ ), grosso $(75 \%$ passando por peneira de malha de $0,15 \mathrm{~mm}$ ), e granulado (entre 1 e $3 \mathrm{~mm}$ de diâmetro). O superfosfato simples, SPS, foi fornecido apenas na forma granulada (entre $1 \mathrm{e}$ $3 \mathrm{~mm}$ de diâmetro). As doses aplicadas foram 50 , 100 e $200 \mathrm{ppm}$, com base no teor total de fósforo dos produtos $(8,2 \%$ de $P$ para as duas fontes).

Foram feitos 7 (sete) cultivos sucessivos: feijão mungo, milho, milho, trigo, milho, milho e trigo. Os periodos de cultivo foram os seguintes, em dias, respectivamente: $15,56,56,150,56,56$, e 150. As plantas (3 por vaso) foram colhidas quando eram visíveis as diferenças entre tratamentos.

Determinou-se a matéria seca produzida e - conteúdo do $\mathbf{P}$ nas plantas após cada cultivo (MALAVOLTA et al., 1989).

Amostras de solo foram coletadas dos vasos depois do terceiro, quarto e sétimo cultivos e nelas determinou-se o $P$ disponível mediante 0 cálculo do valor E (VOSE, 1980), extração por Mehlich 1 e Bray-1 (OLSEN \& DEAN, 1976) e resina (RAIJ \& QUAGGIO, 1983).

\section{RESULTADOS \& DISCUSSÃO}

A TABELA 1 mostra os dados de produção de matéria seca nos vários cultivos. A resposta ao $P$ é evidente e bem assim a diferença entre fontes e doses, o que será discutido em outra publicação. A TABELA 2, que dá o conteúdo de fósforo nas plantas, está de acordo com a TABELA 1: a produção de matéria seca guarda proporcionalidade com o P absorvido.
A variação nos teores de $P$ disponivel avaliados pelos quatro métodos está resumida na TABELA 3. Considerando-se as médias dos teores encontrados em cada extrator e em cada cultivo, independentemente da fonte e da dose, obtém-se os seguintes valores em ppm, respectivamente para $o$ $3^{\circ}, 4^{\circ}$ e $7^{\circ}$; Bray: 27, 27 e 27; Mehlich-1: 19, 19 e 28; resina: 30,26 e 12; valor $E: 87,83$ e 27 . Somente a resina e o valor $\mathrm{E}$, portanto, mostraram um comportamento de acordo com o esperado, isto É, diminuição no teor com o cultivo devido ao consumo do $\mathbf{P}$ do adubo.

Note-se ainda que os valores fornecidos pelo valor $\mathrm{E}$, que supostamente mede apenas o $\mathrm{H}_{2} \mathrm{PO}_{4}^{-}$trocável, excedem os demais. Com o aumento no tempo de incubação de fontes de $\mathrm{P}_{2} \mathrm{O}_{5}$ com o solo em geral nota-se uma diminuição rápida no $\mathbf{P}$ disponível no início e depois uma tendência assintótica (BARROW, 1974; NOVAIS et al., 1990). No presente experimento, devido ao efeito combinado dos cultivos e das reações de fixação, dever-se-ia esperar redução ainda maior o que, entretanto, foi evidenciado somente no caso de dois extratores, a resina e o valor E. Pode-se pensar que tenham se formado no solo fosfatos de $\mathrm{Al}$ e $\mathrm{Fe}$ que os dois extratores ácidos não conseguiram solubilizar devido ao seu $\mathrm{pH}$ baixo.

A TABELA 4 mostra a correlação existente entre os teores de $\mathbf{P}$ disponível ou extraível, como preferem THOMAS \& PEASLEE (1973), segundo pares de extratores. Pode-se ver que somente quando o valor $\mathrm{E}$ é uma das variáveis aparecem coeficientes de correlação não significativos, particularmente quando se usaram dados obtidos depois do $4^{\circ}$ cultivo. As correlações encontradas devem refletir a semelhança entre as formas de $P$ extraídas. A alta correlação dos dados obtidos pela técnica da diluição isotópica e a extração pela resina relatada por ROCHE (1983) não foi encontrada no presente trabalho.

Os teores obtidos com os diferentes extratores se correlacionaram com a produção de matéria seca e com as quantidades de $\mathbf{P}$ absorvido conforme se vê na TABELA 5. Entre matéria seca e $P$ absorvido foi encontrada correlação positiva e significativa a 0,01 , com os coeficientes ( $r$ ) iguais a $0,87,0,99$ e 0,93 , respectivamente para o $3^{\circ}, 4^{\circ}$ e $7^{\circ}$ cultivos. As equações de regressão correspondentes foram: $y=-3,79+2,39 x$, $\mathrm{y}=-0,10+1,00 \mathrm{x}$ e $\mathrm{y}=0,056+1,09 \mathrm{x}$. Segue-se daí que é válido comparar-se os extratores usando os dados de matéria seca ou os do conteúdo de $\mathbf{P}$ das plantas. 
TABELA 1 - Produção de matéria seca em 7 cultivos, com aplicação de fontes e doses de fósforo em um Latossolo.

\begin{tabular}{|c|c|c|c|c|c|c|c|c|}
\hline \multirow[b]{2}{*}{ Fonte } & \multirow{2}{*}{$\begin{array}{r}\text { Dose de } \\
\mathrm{P} \\
\end{array}$} & \multicolumn{7}{|c|}{ Cultivo $^{(1)}$} \\
\hline & & 1 & 2 & 3 & 4 & 5 & 6 & 7 \\
\hline & ppm & & & $\ldots$ & $150^{(2)}$ & & & \\
\hline Yoorin & 0 & 1,2 & 6,60 & 2,89 & 0,24 & 8,49 & 8,93 & 0,52 \\
\hline \multirow[t]{3}{*}{ pó } & 50 & 1,1 & 15,21 & 11,41 & 2,63 & 22,45 & 15,77 & 0,64 \\
\hline & 100 & 1,5 & 22,72 & 22,76 & 2,77 & 27,64 & 27,60 & 0,76 \\
\hline & 200 & 1,4 & 25,57 & 29,14 & 3,83 & 35,99 & 35,56 & 1,34 \\
\hline Yoorin & 50 & 1,3 & 13,38 & 15,20 & 2,58 & 17,82 & 14,17 & 0,68 \\
\hline \multirow[t]{2}{*}{ grosso } & 100 & 1,2 & 19,20 & 19,33 & 4,35 & 27,33 & 23,54 & 0,66 \\
\hline & 200 & 1,4 & 2,51 & 26,64 & 3,98 & 37,70 & 34,43 & 3,02 \\
\hline Yoorin & 50 & 1,1 & 6,02 & 5,03 & 1,57 & 14,10 & 18,55 & 0,86 \\
\hline granu- & 100 & 1,1 & 10,86 & 9,95 & 1,84 & 29,17 & 30,89 & 1,30 \\
\hline lado & 200 & 1,2 & 16,90 & 23,91 & 3,36 & 32,50 & 36,46 & 3,07 \\
\hline \multirow[t]{3}{*}{ SPS } & 50 & 1,5 & 22,09 & 17,35 & 1,89 & 26,76 & 21,78 & 0,77 \\
\hline & 100 & 1,4 & 28,39 & 22,89 & 3,15 & 21,45 & 16,60 & 0,80 \\
\hline & 200 & 1,4 & 35,06 & 37,55 & 3,56 & 38,15 & 14,75 & 1,09 \\
\hline
\end{tabular}

"Cultivos sucessivos de 1 a 7 = feijão mungo, milho, milho, trigo, milho, milho e trigo.

${ }^{2}$ Média de três repetições.

O exame dos valores dos coeficientes de correlação mostra que os valores mais altos aparecem quando se usou resina ou Mehlich 1 como extrator, vindo depois Bray $1 \mathrm{e}$ finalmente o valor E. RAIJ (1978) fez um levantamento exaustivo da relação existente entre teor de $P$ no solo de acordo com diferentes métodos de extração e a resposta à adubação fosfatada. Os coeficientes de determinação mais altos $\left(\mathrm{R}^{2}\right)$, para citar apenas os métodos usados no presente trabalho foram encontrados, em ordem decrescente, com a resina, Mehlich 1, Bray 1 e o valor E.
Os dados reunidos por KAMPRATH \& WATSON (1980) revelaram coeficientes de correlação praticamente iguais quando se comparou a extração por Mehlich e Bray 1 com o conteúdo de $P$ da planta.

ROCHE (1983), trabalhando com solos franceses mostrou que a resina dava valores de $r$ maiores que Bray-1 (Mehlich e valor $E$ não entraram na comparação) quando o conjunto de solos era considerado.

Entretanto, ao separar os solos segundo o seu tipo, os valores obtidos com a resina podiam 
TABELA 2. Fósforo em 7 cultivos, com aplicação de fontes e doses de fósforo em um Latossolo.

\begin{tabular}{|c|c|c|c|c|c|c|c|c|}
\hline \multirow[b]{2}{*}{ Fonte } & \multirow{2}{*}{$\begin{array}{r}\text { Dose de } \\
\mathbf{P}\end{array}$} & \multicolumn{7}{|c|}{ Cultivo (l) } \\
\hline & & 1 & 2 & 3 & 4 & 5 & 6 & 7 \\
\hline & ppm & .... & ......... & ....... & $0^{(2)} \ldots$ & ............ & $\ldots$ & \\
\hline Yoorin & 0 & 2,5 & 5,0 & 2,9 & 1,6 & 8,4 & 9,9 & 0,5 \\
\hline \multirow[t]{3}{*}{ pó } & 50 & 3,2 & 15,2 & 12,5 & 12,5 & 22,4 & 15,5 & 0,6 \\
\hline & 100 & 5,0 & 31,8 & 27,2 & 11,3 & 27,6 & 29,6 & 0,7 \\
\hline & 200 & 7,6 & 53,7 & 52,4 & 23,9 & 36,0 & 41,0 & 1,8 \\
\hline Yoorin & 50 & 2,9 & 14,7 & 22,8 & 7,8 & 17,8 & 15,2 & 0,7 \\
\hline \multirow[t]{2}{*}{ grosso } & 100 & 3,2 & 17,3 & 25,1 & 17,8 & 27,3 & 26,9 & 0,6 \\
\hline & 200 & 3,9 & 27,9 & 37,3 & 13,2 & 37,7 & 45,9 & 2,8 \\
\hline Yoorin & 50 & 2,6 & 3,6 & 4,5 & 3,7 & 14,0 & 21,8 & 0,9 \\
\hline \multirow[t]{2}{*}{ granulado } & 100 & 2,6 & 9,8 & 9,9 & 8,5 & 29,2 & 35,7 & 1,6 \\
\hline & 200 & 3,3 & 15,2 & 31,1 & 15,2 & 32,5 & 40,9 & 2,6 \\
\hline \multirow[t]{3}{*}{ SPS } & 50 & 5,3 & 30,9 & 20,8 & 6,5 & 26,7 & 22,4 & 0,8 \\
\hline & 100 & 5,7 & 53,9 & 25,1 & 7,3 & 21,4 & 14,0 & 1,0 \\
\hline & 200 & 9,1 & 87,6 & 83,0 & 20,2 & 38,2 & 19,3 & 1,7 \\
\hline
\end{tabular}

"Cultivos sucessivos de 1 a 7 = feijão mungo, milho, milho, trigo, milho, milho e trigo.

${ }^{2}$ Média de três repetições.

ser inferiores aos conseguidos com Bray-1 o que, deve refletir as formas de $P$ predominantes em cada um. Resultado semelhante foi relatado por GOEDERT (1981); o extrator de Mehlich 1, por sua alta acidez, solubiliza $\mathrm{P}$ ligado a $\mathrm{Ca}$ e por isso superestima o fósforo disponível em solo tratado com apatita; nessas condições Bray 1 se comportou melhor.

\section{CONCLUSÕES}

A aplicação de superfosfato simples e de termofosfato magnesiano em vasos com amostra de um Latossolo Vermelho Amarelo fase cerrado pro- vocou elevação no teor de $\mathrm{P}$ disponível ou extraível (avaliado com diferentes extratores) que, por sua vez, causaram variações na matéria seca e no conteúdo do elemento nas plantas cultivadas em sucessão. A análise dos dados obtidos permitiu tirar outras conclusões a saber:

(1) a produção de matéria seca ou conteúdo de $P$ mostraram-se variáveis igualmente adequadas para a avaliação da disponibilidade do elemento;

(2) o comportamento dos extratores, resina, Mehlich 1, Bray 1 e o valor E não foi influenciado pelas fontes de $\mathrm{P}_{2} \mathrm{O}_{5}$ usadas; 


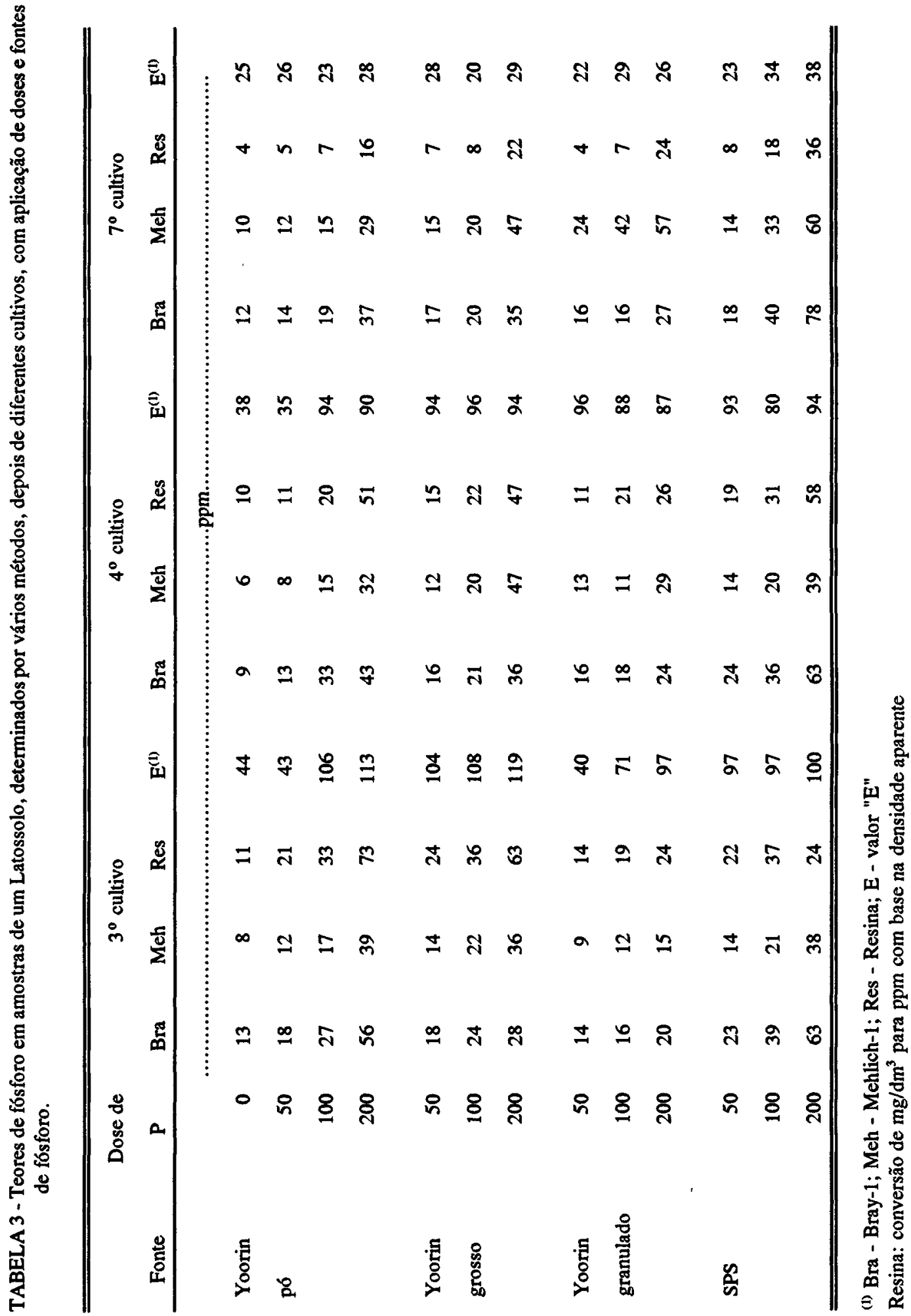


TABELA 4. Correlação entre os teores de $P$ disponível avaliados por diferentes extratores.

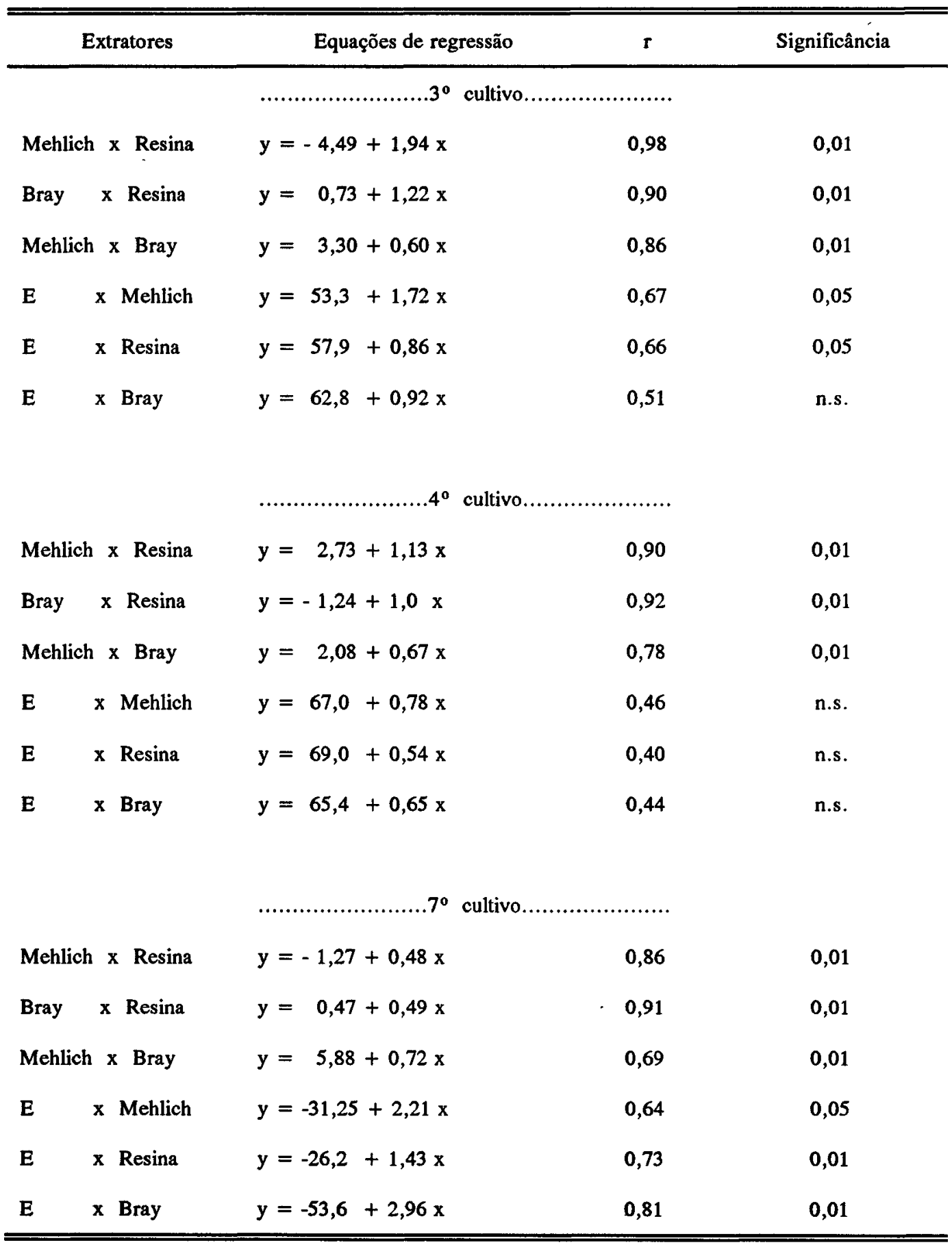


TABELA 5 - Correlações entre P disponível depois do $3^{\circ}, 4^{\circ}$ e $7^{\circ}$ cultivos com a produção de matéria seca e absorvido, respectivamente no $4^{\circ}, 5^{\circ}$ e $7^{\circ}$ cultivos.

\begin{tabular}{|c|c|c|c|}
\hline Extratores & Equações de regressão & $\mathbf{r}$ & Significância \\
\hline & \multicolumn{3}{|c|}{ 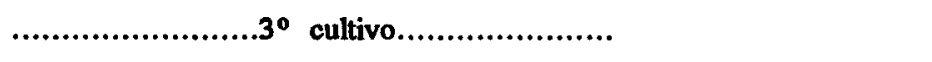 } \\
\hline Resina $\times$ m. seca & $y=3,2+0,09 x$ & 0,81 & 0,01 \\
\hline Mehlich X m. seca & $y=2,9+0,17 x$ & 0,80 & 0,01 \\
\hline E $\quad$ x m. seca & $y=0,77+0,06 x$ & 0,75 & 0,01 \\
\hline Bray $\mathrm{x}$ m. seca & $y=3,40+0,10 x$ & 0,72 & 0,05 \\
\hline Resina $x$ P. abs. & $y=3,15+0,24 x$ & 0,80 & 0,01 \\
\hline Mehlich X P. abs. & $y=2,13+0,46 x$ & 0,78 & 0,01 \\
\hline E $\quad$ X P. abs. & $y=0,49+0,13 x$ & 0,58 & 0,05 \\
\hline Bray $\times$ P. abs. & $y=3,28+0,29 x$ & 0,72 & 0,01 \\
\hline \multicolumn{4}{|c|}{. cultivo............................................... } \\
\hline Resina $\times$ m. seca & $y=13,9+0,46 x$ & 0,81 & 0,01 \\
\hline Mehlich X m. seca & $y=14,1+0,57 x$ & 0,81 & 0,01 \\
\hline E $\quad$ x m. seca & $y=8,4+0,21 x$ & 0,48 & n.s. \\
\hline Bray $\mathrm{x}$ m. seca & $y=13,4+0,46 x$ & 0,74 & 0,01 \\
\hline Resina $\times$ P. abs. & $y=13,8+0,46 x$ & 0,81 & 0,01 \\
\hline Mehlich X P. abs. & $y=14,0+0,58 x$ & 0,81 & 0,01 \\
\hline E $\quad$ x P. abs. & $y=8,3+0,21 x$ & 0,81 & 0,01 \\
\hline Bray x P. abs. & $y=13,4+0,46 x$ & 0,74 & 0,01 \\
\hline \multicolumn{4}{|c|}{. } \\
\hline Resina $x \mathrm{~m}$. seca & $y=0,54+0,05 x$ & 0,56 & 0,05 \\
\hline Mehlich $\mathrm{x}$ m. seca & $y=0,13+0,03 x$ & 0,72 & 0,01 \\
\hline E $\quad$ m. seca & $y=0,44+0,02 x$ & 0,14 & n.s. \\
\hline Bray $x \mathrm{~m} . \operatorname{seca}$ & $y=0,90+0,01 x$ & 0,20 & n.s. \\
\hline Resina $x$ P. abs. & $y=0,49+0,06 x$ & 0,64 & 0,05 \\
\hline Mehlich X P. abs. & $y=0,06+0,04 x$ & 0,75 & 0,01 \\
\hline E $\quad$ X P. abs. & $y=-0,51+0,06 x$ & 0,33 & n.s. \\
\hline Bray X P. abs. & $y=0,75+0,02 x$ & 0,40 & n.s. \\
\hline
\end{tabular}


(3) as correlações mais altas entre $P$ disponível $e$ matéria seca produzida ou fósforo absorvido foram verificadas com os dados obtidos quando se usou os extratores resina, Mehlich 1, vindo depois Bray 1 e, por último, o valor $\mathrm{E}$.

\section{REFERENCIAS BIBLIOGRÁFICAS}

ANGHINONI, I.; VOLKWEISS, S.J. Recomendaçōs de uso de fertilizantes no Brasil. In: SIMPOSIO SOBRE FERTILIZANTES NA AGRICULTURA BRASILEIRA,. 1984, Brasilia. Anais... Brasília: EMBRAPA/ DEP, 1984. p. 179-204.

BARROW, N.J. The slow reactions between soil an anions. I. Effect of time, temperature and water content of a soil on the decrease in effectiveness of phosphate for plant growth. Soil Science, Baltimore, v.118, p.380-386. 1974.

GOEDERT, W. Resposta agronómica do cerrado ao uso do fosfato. In: ENCONTRO NACIONAL DE ROCHA FOSFÁTICA, 2., 1981 . Brasília, São Paulo: IBRAFOS. 1981 . p.262-274.

KAMPRATH, E.J.; WATSON, M.E. Conventional soil and tissue tests for assessing the phosphorus status of soils. In: KHASAWNEH, F.E.; SAMPLE, E.C.; KAMPRATH, E.J., ed. The role of phosphorus in agriculture. Madison: American Society of Agronomy, 1980, cap.16, p.433-470.

MALAVOLTA, E.; VITTI, G.C.; OLIVEIRA, S.A. Avaliaça do estado nutricional das plantas: princfpios e aplicaçoes. Piracicaba: Potafós, 1989. $201 p$.

MCAULIFFE, C.D.; HALL, N.S.; DEAN, L.A.; HENDRICKS, S.B. Exchange reaction between phosphate and soil minerals. Soil Science Society of America Proceeding, Baltimore v.12., p.119-123. 1948.

NOVAIS, R.F.; NEVES, J.C.L.; BARROS, N.F. Aspectos físico-químicos envolvidos na fixação do fósforo no solo. In: ENCONTRO NACIONAL DE ROCHA FOSFÁTICA, 5., 1990, São Paulo. São Paulo: IBRAFOS. 1990. p.132-164.
OLSEN, S.R.; DEAN, L.A. Phosphorus. In: BLACK, C.A., ed. Methods of soil analysis, Part 2, Chemical and Microbiological properties. Madison: American Society of Agronomy, 1976. cap.3, p.1035-1049.

RAIJ, B. van. Seleção de métodos de laboratório para avaliar a disponibilidade de fósforo em solos. Revista Brasileira de Ciencia do Solo, Campinas, v.2, p.1-9, jan/abr. 1978.

RAJ, B. Van; QUAGGIO, J.A.; SILVA, N.M. Extraction of phosphorus, potassium, calcium and magnesium from soils by an ion-exchange resin procedure. Communications in Soil Science and Plant Analysis, New York, v.17, p.547-556. 1986.

RAIJ, B. Van; QUAGGIO, J.A. Métodos de analise de solo para fins de fertilidade. Campinas: Instituto Agronômico, 1983. 31p. (Boletim Técnico, 81).

ROCHE, P. Les methodes d'appreciation du statut phosphorique des sols. Leur application à l'estimation des besoins en engrais phosphatés. In: CONGRES INTERNATIONAL SUR LES COMPOSES PHOSPHORÉ, 3., 1983. Bruxelas. Actes... Casablanca: Institut du Phosphate, 1984. p.165-194.

SMITH, F.W.; ELLIS, B.G.; GRAVA, J. Use of acidfluoride solutions for the extraction of available phosphorus in calcareous soils and in soils to which rock phosphate has been added. Soil Science Society of American Proceedings, Baitimore, v.21, p.400404. 1957.

THOMAS, G.W.; PEASLLE, D.E. Testing soil for phosphorus. In: WALSH, L.M.; BEATON, J.D., ed. Soil Testing and Plant Aualysis. Madison: SSSA, 1973, p.115-32.

VOSE, P.B. Introduction to nuclear techniques in agronomy and plant biology. Oxford: Pergamon Press, 1980. 391 p.

Enviado para publicação em 03.06 .93

Aceito para publicação em 13.12 .93 